\title{
Osteoarticular tuberculosis in an HIV-positive patient: a case report
}

\author{
Gabriela de Moraes Rêgo Guedes ${ }^{[1],[2]}$, Juliana Figueirêdo da Costa Lima ${ }^{[2],}$ \\ Fabiana Cristina Fulco Santos ${ }^{[2]}$, Marcela Pereira Salazar ${ }^{[2]}$, Klarissa Miranda Guarines ${ }^{[2]}$, \\ Lílian Maria Lapa Montenegro ${ }^{[2]}$, Ivan da Rocha Pitta ${ }^{[3]}$ \\ and Haiana Charifker Schindler ${ }^{[1],[4]}$
}

[1]. Centro de Ciências Biológicas, Universidade Federal de Pernambuco, Recife, PE. [2]. Laboratório de Imunoepidemiologia, Departamento de Imunologia, Centro de Pesquisas Aggeu Magalhães, Recife, PE. [3]. Departamento de Farmácia, Universidade Federal de Pernambuco, Recife, PE. [4]. Departamento Materno Infantil, Universidade Federal de Pernambuco, Recife, PE.

\begin{abstract}
The authors report a case of a 38-year-old HIV-positive woman, with subcutaneous nodules on the thoracic region with 3 months of evolution. Clinical, laboratory, and epidemiological features were evaluated and associated with apparent damage to the T11-T12 vertebrae, identification by imaging tests, positivity in a polymerase chain reaction-based test, and reactivity to the Mantoux tuberculin skin test (PPD-RT 23). The patient was diagnosed with osteoarticular tuberculosis and received treatment for a year, and clinical cure was achieved.
\end{abstract}

Keywords: Osteoarticular tuberculosis. PCR. Diagnosis.

\section{INTRODUCTION}

Tuberculosis (TB) remains one of the most devastating and widespread infections in the world. In 2010, 71,000 cases were reported in Brazil, making TB the most common cause of death in HIV patients $\mathrm{HIV}^{1}$. In HIV-infected individuals, over $50 \%$ of TB cases are extrapulmonary ${ }^{2}$. The skeletal form of TB affects $1-3 \%$ of patients diagnosed with extrapulmonary tuberculosis (EPTB), affecting the spine in $50 \%$ of cases $^{3}$.

Osteoarticular TB diagnosis is most commonly based on clinical and radiological features. New molecular diagnostic tests have been developed, such as the polymerase chain reaction (PCR), that detect unique nucleotide sequences of Mycobacterium tuberculosis, mainly for the paucibacillary forms. PCR-based tests can generate results in 24-72 $\mathrm{h}$ and offer higher accuracy than conventional methods, such as bacilloscopy and culture ${ }^{4}$. The aim of this case report was to show how the new techniques were applied towards the final disease diagnosis in the case of an human immunodeficiency virus (HIV)-positive patient diagnosed with skeletal TB.

Address to: Dr ${ }^{\mathrm{a}}$ Gabriela Moraes Rêgo Guedes. Centro de Ciências Biológicas/ UFPE. Av. Professor Moraes Rego, s/n, Cidade Universitária, 50670-400 Recife, PE, Brasil.

Phone: 5581 2101-2569

e-mail: gabrielamrg31@hotmail.com

Received 21 October 2013

Accepted 22 January 2014

\section{CASE REPORT}

A 38-year-old female patient who was from Vitória, Pernambuco, Brazil, had been diagnosed with HIV in 1995 , and had a previous history of neurotoxoplasmosis and TB (ganglionic and pleural) was treated with antiretroviral therapy (azithromycin + lamivudine + efavirenz) beginning in 2009 . The patient presented at the Clinics' Hospital in Pernambuco in November of 2011 with subcutaneous nodules on the sternal region that had persisted for 3 months, following sudden appearance without any associated signs or symptoms. Nodule aspiration was performed and histopathological results showed granulomatous chronic inflammation with the presence of giant Langerhans cells and foreign body materials, negative results for acid-fast bacilli (AFB) and fungus, and absence of neoplasia. Following aspiration of the nodule, a fistulous orifice was revealed with seropurulent secretion drainage, without spontaneous healing,

A chest X-Ray revealed an osteolytic lesion on the thoracolumbar segment of the vertebrae. Magnetic resonance imaging (MRI) of the thoracic spine showed irregular and insufflated bone lesions, compromising the lateral-right aspect of the thoracic vertebral body (lower part of T11 and superior of T12). The MRI also revealed slight hypointense lesions on T1's weighted sequences (Figure 1) and heterogeneous signs on the T2 vertebra (Figure 2) with impregnation of the intravenous contrast medium, delineating central areas of necrosis/liquefaction. Bone densitometry testing identified foci of osteopenia in the femoral neck and in the L1-L4 vertebrae. Ultrasonography of the chest wall showed an elongated image, 


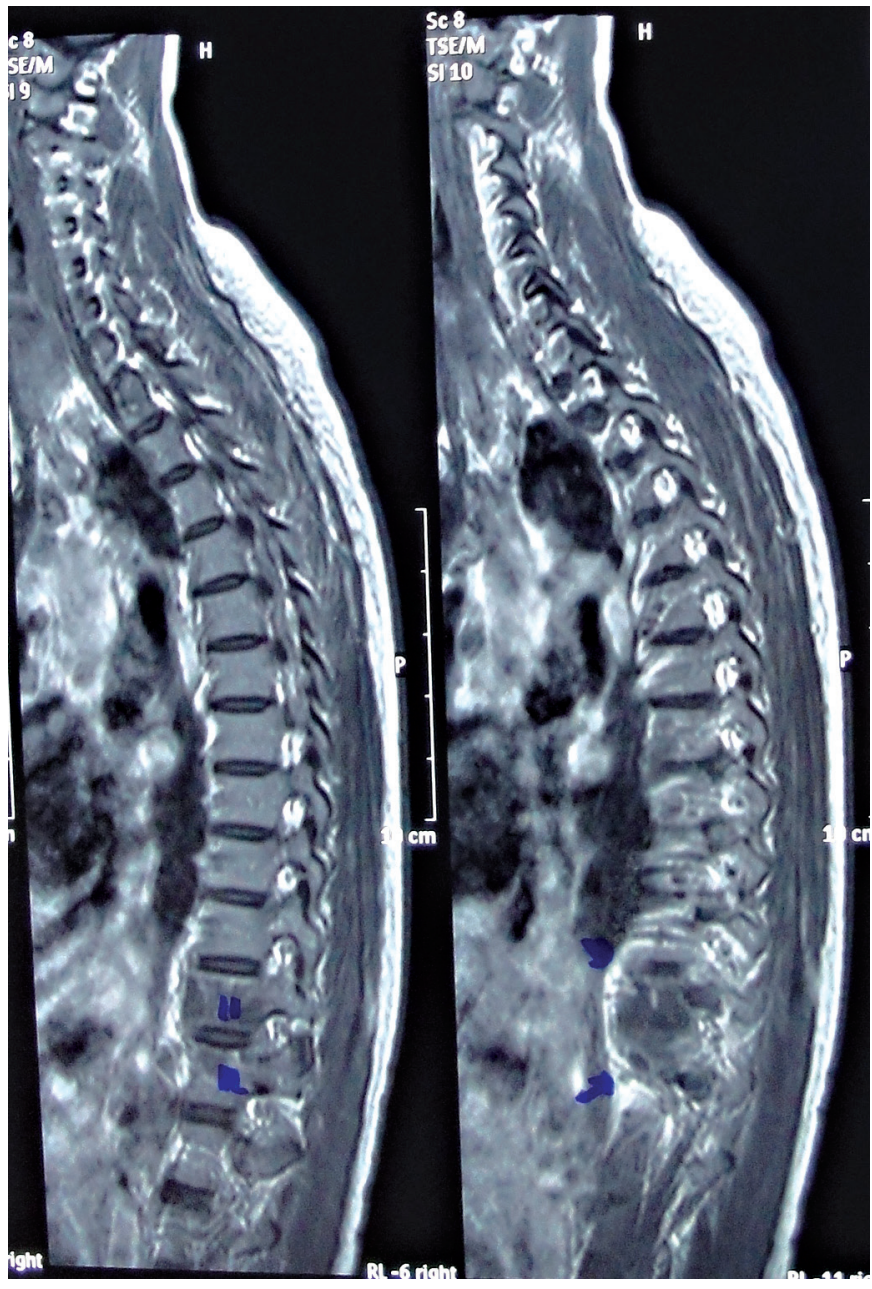

FIGURE 1 - Magnetic resonance imaging showing lesions of the T11 and T12 vertebral bodies.

situated on the medium line and below the subcutaneous plan, extending anteriorly to the sternum and the medial region of the left breast, measuring $8.5 \times 2.5 \times 0.7 \mathrm{~cm}$, with a volume of $7.7 \mathrm{~mL}$, presenting a fistulous path to the skin.

The tuberculin test (Mantoux test) was performed in a $20 \mathrm{~mm}$ reaction. The patient did not show the Bacillus Calmette-Guérin (BCG) vaccine scar. The Single Tube Nested-PCR (STN-PCR) test was positive for M. tuberculosis in blood and urine samples, although its culture on Lowenstein-Jensen medium was negative. Negative results were also obtained using the QuantiFERON-TB Gold In-Tube Assay. The patient's Cluster of differentiation $4(\mathrm{CD} 4+) \mathrm{T}$ cell count was 528 cells $/ \mu \mathrm{L}$. The diagnosis of chronic osteomyelitis resulting from osteoarticular TB was confirmed after 2 months of clinical investigation, in agreement with the positive results of the Mantoux and STN-PCR tests. Thus, a treatment regimen for TB with rifampicin, isoniazid, pyrazinamide, and ethambutol was initiated for 2 months, followed by 4 months of isoniazid and rifampicin treatment. The patient achieved a therapeutic cure at the end of the treatment.

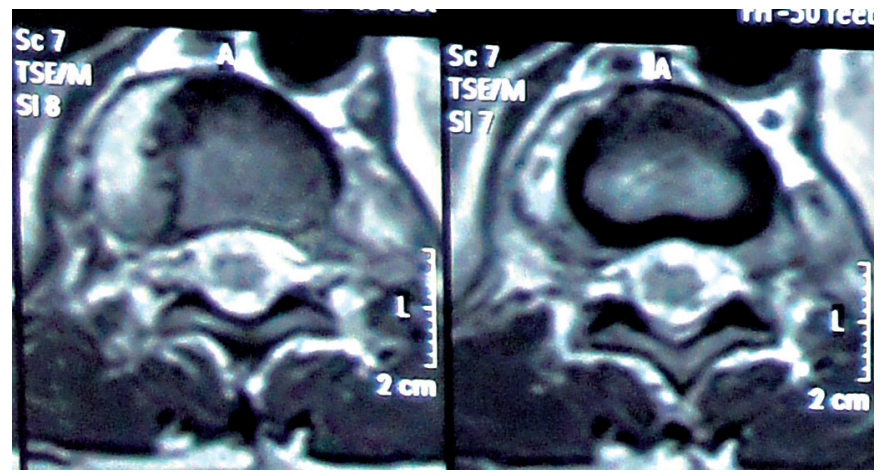

FIGURE 2 - Magnetic resonance imaging showing areas of necrosis and liquefaction.

\section{DIscussion}

The signs and symptoms of osteoarticular TB are frequently nonspecific and similar to those of several infectious and non-infectious diseases, such as rheumatoid arthritis, septic arthritis, chronic osteomyelitis and bone methastasis ${ }^{5}$. In a series of cases, $31.5 \%$ of patients with skeletal TB did not have systemic symptoms ${ }^{5}$. The characteristics of TB associated with HIV include extrapulmonary disease, disseminated disease, fast progression, visceral lymphadenopathy, tissue abscesses, and a negative tuberculin test ${ }^{6}$. The HIV-positive patient discussed in this case study did not present any common signs of pulmonary TB, but reacted to the Mantoux test and presented a fistulous orifice after aspiration of a subcutaneous nodule.

Osteoarticular TB normally begins as osteomyelitis on the bone growth plates where the blood supply is plentiful, and then branches out to infiltrate less vascularized, local spaces. On the vertebrae, the most frequent segment that is infected by M. tuberculosis is the thoracic spine. Infection begins on the anteroinferior aspect of the vertebral body, with destruction of the intervertebral disc and the adjacent vertebrae. Abscesses with extensions to the surface or adjacent tissues may be found ${ }^{6}$. The stricken segments of this patient were the thoracic vertebrae T11 and T12, as detected by MRI.

The diagnosis of osteoarticular TB is unspecific and the disease is frequently long lasting, persisting on average for 12 to 19 months and lasting up to 10 years, which is likely due to the insidious presentation of the disease and a low index of suspicion regarding the diagnosis ${ }^{5}$. Compared with the average time of skeletal TB diagnosis reported in the literature, a diagnosis accomplished in only 2 months is considered fast for an extrapulmonary TB case. The medical staff involved compared the standard Mantoux test, considered a flawed technique for use with immunosuppressed patients, to the innovative STNPCR assay, which was positive for blood and urine samples.

MRI is the imaging test of choice for vertebral TB because it allows practitioners to differentiate tissues from granulation and abscess, to evaluate the rate of bone destruction, and to identify 
the invasion of adjacent structures, such as the bone marrow ${ }^{6}$. However, anatomy and bone abnormalities, including gross calcifications are better observed by computed tomography scanning. Therefore, it is important to compare the 2 image methods to confirm the diagnosis.

It is recommended that EPTB be diagnosed bacteriologically, histopathologically, or by the clinical evaluation of specialists. In most cases, clinical diagnosis is performed and the empiric therapeutic answer is frequently used to confirm the diagnosis? PCR is a highly efficient and rapid method for diagnosing diseases, making this approach of great value in early diagnoses. As PCR is a very sensitive technique that can detect 1 or 2 mycobacteria in a sample, treatment can begin immediately based on PCR results if there are clinical signs of the disease. Although PCR results cannot replace bacilloscopy, culture, or histopathological observations, they can contribute significantly to the early diagnosis of EPTB and have an acceptable impact on the clinical management of the disease ${ }^{8}$.

The patient showed a previous history of TB that may have been the originating factor for the new diagnosis. One remaining granulomatous focus contained a latent infection that may have been reactivated by a decrease in immunity. Similar to the case reported by our team, Kakarala and Rajan ${ }^{9}$ have also described a case related to osteoarticular TB, which affected the ankle, where the patient had a previous history of TB.

Payne and Yang ${ }^{10}$ reported a case of osteoarticular TB in a 40-year-old malnourished patient, who presented with an ulcerative lesion of about $3 \times 4 \mathrm{~cm}$ in the posterolateral face of the right knee, which continuously produced a milky secretion. This patient underwent the Mantoux test and presented an induration of $21 \mathrm{~mm}$. This report shares common features with the case in discussion: a fistulous orifice, which drained a secretion, and a reactive tuberculin test. While a positive Mantoux test result can be useful for confirming a suspicion of TB, a negative Mantoux test result cannot exclude TB.

Knowing that TB can affect many organs besides the lungs, it is fundamental to evaluate the involvement of extrapulmonary sites $^{10}$. TB should be considered as a possible diagnosis, especially when patients exhibit immunosuppression or come from an endemic area, such as Brazil.

To establish the diagnosis of skeletal TB, it is necessary to have a high index of suspicion. Practitioners must consider the skeletal TB in patients with an inconclusive clinical course, presenting osteomyelitis involving the thoracic spine, even when bacterial cultures are negative. It is important to employ the use of additional tools for diagnosing TB. In the present case, the molecular technique of STN-PCR was more accurate than conventional culture, the gold standard used for TB diagnosis, which showed negative growth of bacilli with sowed urine. The PCR amplification technique is also more sensitive than the histopathological method, wherein no acid-fast bacilli were found with the analyzed sample.

\section{ACKNOWLEDGMENTS}

The authors thank Drs. Fabiana Gonzaga and Renata Florêncio from the Infectious and Parasitic Diseases' Service of the Clinic's Hospital at the Federal University of Pernambuco for their assistance in this study.

\section{FINANCIAL SUPPORT}

Programa de Desenvolvimento Tecnológico em Insumos para Saúde (PDTIS) / Fundação Oswaldo Cruz (FIOCRUZ) and Conselho Nacional de Desenvolvimento Científico e Tecnológico $(\mathrm{CNPq})$

\section{REFERENCES}

1. Ministério da Saúde. Tratamento Diretamente Observado (TDO) da Tuberculose na Atenção Básica. Protocolo de Enfermagem. Brasil: Ministério da Saúde; 2011.

2. Cortez MV, Oliveira CM, Monte RL, Araújo JR, Braga BB, Reis DZ, et al. HIV associated tuberculous lymphadenitis: the importance of polymerase chain reaction (PCR) as a complementary tool for the diagnosis of tuberculosis - a study of 104 patients. An Bras Dermatol 2011; 86:925-931.

3. Sun YS, Lou SQ, Wen JM, Lv WX, Jiao CG, Yang SM, et al. Clinical value of polymerase chain reaction in the diagnosis of joint tuberculosis by detecting the DNA of Mycobacterium tuberculosis. Orthop Surg 2011; 3:64-71.

4. Haldar S, Bose M, Chakrabarti P, Daginawala HF, Harinath BC, Kashyap RS, et al. Improved laboratory diagnosis of tuberculosis - the Indian experience. Tuberculosis 2011; 91: 414-426.

5. Haas DW. Mycobacterium tuberculosis. In: Mandel GL, Bennett JE, Dolin R, editors. Principles and Practice of Infections Diseases, $5^{\text {th }}$ edition, Churchill Livingstone; 2000; p. 2576-2607.

6. Canadian Thoracic Society. Canadian tuberculosis standards. $5^{\text {th }}$ edition Ottawa: Health Canada; 2000. p. 4. Cat n ${ }^{\circ}$ H49-146/2000E.

7. Golden MP, Vikram HR. Extrapulmonary tuberculosis: an overview. Am Fam Physician 2005; 72:1761-1768.

8. Mehta PK, Raj A, Singh N, Khuller GK. Diagnosis of extrapulmonary tuberculosis by PCR. FEMS Immunol Med Microbiol 2012; 66:20-36.

9. Kakarala G, Rajan D. Rare presentation of osteoarticular tuerculosis. Acta Orthop Belg 2005; 71:626-627.

10. Payne K, Yang J. Osteoarticular tuberculosis: a case report and discussion. JAMC 2002; 166:628-630. 